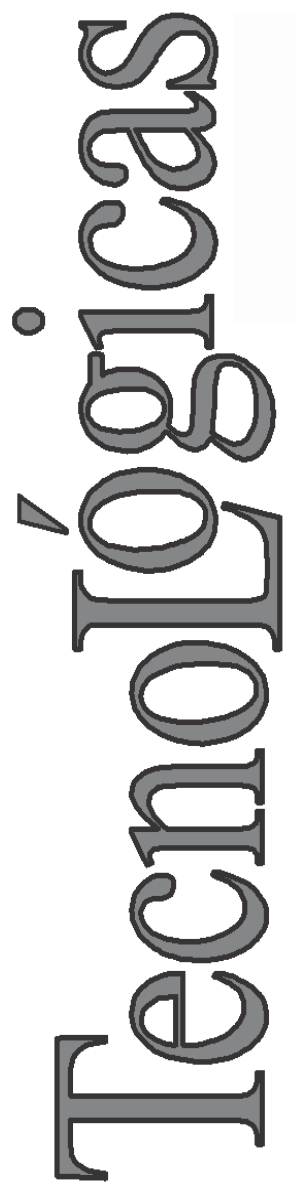

\title{
Dimensionamiento de Sistemas de Generación Fotovoltaicos Localizados en la Ciudad de Medellín
}

\author{
Dimensioning of Photovoltaic \\ Generation Systems Located in \\ Medellin City
}

Laura Herrera ${ }^{1}$

Alina Miranda ${ }^{2}$

Eliana I. Arango-Zuluaga ${ }^{3}$

Carlos A. Ramos-Paja ${ }^{4}$ Daniel González-Montoya ${ }^{5}$

1 Facultad de Minas, Departamento de Energía Eléctrica y Automática, Universidad Nacional de Colombia sede Medellín, Medellín-Colombia lherrera@unal.edu.co

2 Facultad de Minas, Departamento de Energía Eléctrica y Automática, Universidad Nacional de Colombia sede Medellín, Medellín-Colombia asmirandam@unal.edu.co

3 Facultad de Minas, Departamento de Energía Eléctrica y Automática, Universidad Nacional de Colombia sede Medellín, Medellín-Colombia eiarangoz@unal.edu.co

4 Facultad de Minas, Departamento de Energía Eléctrica y Automática, Universidad Nacional de Colombia sede Medellín, Medellín-Colombia caramosp@unal.edu.co

5 Facultad de Minas, Departamento de Energía Eléctrica y Automática, Universidad Nacional de Colombia sede Medellín, Medellín-Colombia dgonzalezm@unal.edu.co 


\title{
Resumen
}

Los sistemas fotovoltaicos son una de las soluciones más eficientes a la creciente crisis energética de las últimas décadas. La implementación de estos sistemas será viable económicamente en la mayoría de los casos, debido a extensa cantidad de ventajas que estos exhiben, sin embargo, en aras de un mejor rendimiento económico, se generan diferentes estrategias para el manejo de estos. El presente artículo muestra una estrategia de optimización basada en la redistribución de energía generada, que busca dejar de consumirla conforme se produce, para aprovecharla en aquellos momentos en los cuales las tarifas de energía son mayores, haciendo uso de un sistema de almacenamiento. Se presentan los pasos para el diseño de un sistema fotovoltaico con dichos criterios y un análisis económico que dicte las condiciones para la viabilidad del mismo.

\section{Palabras clave}

Energías renovables; paneles fotovoltaicos; sistema de almacenamiento; tarifa energética; rentabilidad.

\begin{abstract}
Photovoltaic systems are one of the most efficient solutions to the energy crisis of the last decades. The implementation of these systems will be economically feasible in most cases, due to extensive number of advantages that they exhibit. However, for the sake of better economic performance, it is important to generate different strategies for their management. This paper presents an optimization strategy based on the redistribution of power generated, which seeks to stop spending as it is produced to take advantage of those times when energy rates are higher, making use of a storage system. The steps for the photovoltaic system design with these criteria and economic analysis will be presented.
\end{abstract}

\section{Keywords}

Renewable energy; photovoltaic panels; storage system; energy rate; profitability. 


\section{INTRODUCCIÓN}

La generación de energías limpias o energías verdes, se ha convertido en una necesidad mundial debido a la creciente demanda energética que agota vertiginosamente los recursos utilizados para la generación de energías provenientes de combustibles fósiles (Kateeb, 2011).

Es por lo anterior que las investigaciones acerca de la generación de energía a partir de fuentes alternativas, como la radiación solar, se intensifican de manera especial en las últimas décadas, teniendo en cuenta que además, anualmente la cantidad de energía que se recibe del Sol se estima del orden de 149 millones de KWh, cantidad muy superior al consumo mundial de energía, se hace necesario cambiar progresivamente el paradigma de la generación de energía. El problema radica entonces, en convertir y distribuir con la mayor eficiencia posible dicha radiación en energía eléctrica. (McLauchlan \& Mehrubeoglu, 2010).

Como es evidente, no se puede modificar el período de tiempo en el que los paneles reciben energía, pues esto depende exclusivamente de factores externos como la temperatura y la radiación solar, sin embargo, es posible alterar los momentos en los cuales se consume dicha energía para alcanzar mayor rentabilidad.

Tradicionalmente la energía que producen los sistemas fotovoltaicos es consumida conforme se produce, en el presente artículo se desea mostrar una alternativa para su uso, que pueda significar un aumento en el ahorro de energía que representa su implementación, buscando por consiguiente, un aumento en la rentabilidad económica del sistema. Dicha alternativa se basa en la redistribución de la energía generada según las fluctuaciones tarifarias en diferentes horas del día, para lo cual se hará uso de un sistema de almacenamiento.

\section{2. cálculo de las características del Panel FOTOVOLTAICO}

Se presentará a continuación una metodología para el dimensionamiento de sistemas fotovoltaicos, basada en las característi- 
cas propias de cada panel ajustadas a las exigencias de cada sitio en particular.

Del trabajo de Petrone \& Ramos-Paja (2011), se obtienen los datos base para el desarrollo de esta metodología, concretamente la potencia nominal y las dimensiones de cada panel. La relación entre ellas dicta la eficiencia y por tanto la cantidad de energía que será capaz de producir el sistema. Inicialmente, se requiere calcular la eficiencia de cada panel, para ello se relaciona la potencia que entregaría el panel en condiciones ambientales normalizadas (Temperatura de funcionamiento de $25^{\circ} \mathrm{C}$ y una radiación solar de $1.000 \mathrm{~W} / \mathrm{m}^{2}$ ), potencia ideal, y la potencia nominal, así:

Potencia ideal $[\mathrm{W}]=$ AreaPanel $\left[\mathrm{m}^{2}\right] * 1000\left[\frac{\mathrm{W}}{\mathrm{m}^{2}}\right]$

Eficiencia $[\%]=\frac{\text { Potencia Nominal }[\mathrm{W}]}{\text { Potencia Ideal }[\mathrm{W}]} * 100$

La Eficiencia del panel es un factor que representa su capacidad para transformar la irradiación recibida en potencia eléctrica. Luego, la potencia entregada por cada panel es el producto entre su área, el perfil de irradiación característico de la zona de implementación (Los perfiles de carga se presentan para un día cada hora) y su eficiencia, así: (i representa cada hora del día)

Potencia Entregada(i)[W]

$$
\begin{aligned}
& =\text { AreaPanel }\left[\mathrm{m}^{2}\right] * \operatorname{Irradiación}(\mathrm{i})\left[\frac{\mathrm{w}}{\mathrm{m}^{2}}\right] \\
& * \text { Eficiencia }[\%]_{\mathrm{i}=1,2, \ldots 24}
\end{aligned}
$$

El anterior cálculo arrojará un vector donde cada uno de sus componentes representa la potencia promedio entregada por el panel en la hora $i$. Es necesario expresar dicha potencia en unidades de energía para facilitar cálculos posteriores y estimación costos. Se sabe que las unidades de la energía eléctrica son [Wh], la conversión deseada se logra tomando el valor puntual de potencia entregada y distribuyéndolo en toda la hora. 
$\sum_{i=1}^{24}$ Energia de un panel(i)

Como resultado, se obtiene el perfil de energía eléctrica producido por el panel específico.

\section{DIMENSIONAMIENTO DEL SISTEMA FOTOVOLTAICO}

Habiendo obtenido el perfil de energía generado por cada uno de los paneles, es posible calcular fácilmente la dimensión de todo el sistema, en cuanto a número de paneles y cantidad de energía producida, lo cual estará delimitado por el área disponible para la instalación del arreglo y/o la cantidad de energía que se desea suplir. Suponiendo que el alcance del sistema está delimitado por el área disponible, a partir de esta pueden hacerse estimaciones de Número de paneles y la Energía Suplida por estos, así:

$\#$ Paneles $=\left\lceil\frac{\text { AreaDisponible }\left[\mathrm{m}^{2}\right]}{\text { AreaPanel }\left[\mathrm{m}^{2}\right]}\right\rceil$

Energía Suplida $[\mathrm{Wh}]=$ \#Paneles $* \sum_{\mathrm{i}=1}^{24}$ Energía 1 panel(i)

Si por el contrario, se desea suplir una cantidad específica de energía, será necesario estimar la cantidad de paneles requerida y el área ocupada por dichos paneles; es importante tener en cuenta que si no se dispone del área suficiente para esa cantidad de energía, se deberá replantear dicho valor,

$\#$ Paneles $=\left\lceil\frac{\text { Energía Suplida }}{\sum_{\mathrm{i}=1}^{24} \text { Energía 1panel(i) }}\right\rceil$

Area Ocupada $\left[\mathrm{m}^{2}\right]=$ \#Paneles $*$ AreaPanel $\left[\mathrm{m}^{2}\right]$ 


\subsection{Dimensionamiento de Sistemas Aplicado a los Perfiles de Carga Típicos de Diferentes Sectores en la Ciudad de Medellín}

Cada consumidor de energía eléctrica posee un perfil de carga, que es un gráfico de la variación en carga eléctrica contra el tiempo (UNAL, 2007), es decir, representa el consumo diario dividido en cada hora, usualmente presentado en [KWh]. Establecimientos con propósitos afines pueden presentar perfiles de carga similares, así por ejemplo, dos hospitales diferentes, con diferente cantidad de consumo diario, puede presentar perfiles comparables según las horas de mayor o menor consumo durante el día.

A continuación, como ilustración de todos los procedimientos expuestos anteriormente se desarrolla un diseño para una implementación de un sistema fotovoltaico. Se desea entonces, realizar el montaje de un sistema fotovoltaico para un restaurante en la ciudad de Medellín, cuyo consumo al día es aproximadamente 294 KWh, se pretende suplir un $20 \%$ de la energía que se consume diariamente y dispone de un área total para el montaje de $50 \mathrm{~m}^{2}$.

Se realizará el diseño tomando como referencia el panel BP585, que tiene una potencia nominal de $85 \mathrm{~W}$ y un área de $0.634 \mathrm{~m}^{2}$ según su documentación.

\subsubsection{Características del panel BP585}

Reemplazando el área del panel en (1) se obtiene,

Potencia ideal $=634 \mathrm{~W}$

Reemplazando el valor de la potencia ideal (9) en (2) se obtiene la eficiencia del panel,

Eficiencia $=13.4 \%$

Se requiere ahora, el perfil de irradiación típico en la ciudad de Medellín (Dado por un vector de 24 posiciones en donde cada posición representa una hora del día), con el fin de obtener el perfil de la potencia entregada por un panel BP 585 en 1 día. 


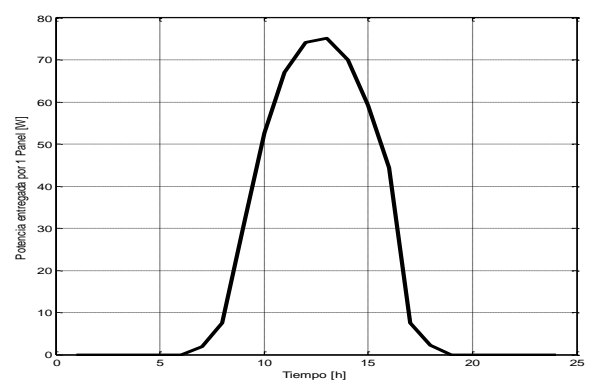

Fig. 1. Perfil de energía recolectada por un panel en un día

Realizando una sumatoria de los valores presentados de cada hora, se obtiene la potencia entregada por el panel BP 585 en 1 día,

Potencia Entregada Dia $=492.4050 \mathrm{~W}$

Energía Entregada Dia $=0.492 \mathrm{KWh}$

\subsubsection{Dimensionamiento del sistema fotovoltaico}

Habiendo calculado todas las características del panel elegido, es posible ahora determinar las del sistema. Se sabe que se desea suplir un $20 \%$ de la energía consumida en un día, es decir 58.8 KWh, reemplazando (12) en (7) se obtiene:

Número de Paneles $=120$

Ahora es necesario calcular el área ocupada por este número de paneles, para encontrar si es posible implementar el sistema con dichos requerimientos. Reemplazando el área del panel y (13) en (8) se obtiene que,

AreaOcupada $=75.5 \mathrm{~m}^{2}$

Se puede observar claramente que el espacio disponible para el montaje no puede albergar el número de paneles necesarios para suplir esa cantidad de energía. Se procede entonces a delimitar el sistema de acuerdo al área disponible, a partir de esta se halla la 
cantidad de paneles. Reemplazando en (5) los valores de área disponible y área del panel, se obtiene,

\#Paneles $=79$

EnergíaSuplida $=38.9 \mathrm{KWh}$

Lo que equivale al $13.3 \%$ del consumo total de energía diario del establecimiento estudiado

\subsubsection{Cálculo de sistema de almacenamiento}

Como se estableció previamente, el objetivo principal del presente artículo es describir una metodología para redistribución de la energía producida por los paneles fotovoltaicos; inicialmente es necesario establecer los periodos de almacenamiento y consumo, que están directamente relacionados con las tarifas de energía, presentadas por la empresa prestadora de servicios de la zona en la que se desea realizar la implementación (EPM, 2011). En el caso específico de la ciudad de Medellín, Empresas Públicas de Medellín . (EPM) establece un plan tarifario en el que los periodos comprendidos entre las $9 \mathrm{am}-12 \mathrm{~m}$ y las $6 \mathrm{pm}-9 \mathrm{pm}$ representan las horas pico de tarifas. Se sabe igualmente, que la irradiación solar y por tanto el periodo en el que los paneles producen energía está comprendido entre las $7 \mathrm{am}$ y las $6 \mathrm{pm}$, deberá redistribuirse esa energía producida durante este periodo, de tal manera que sea aprovechada en las horas pico tarifarias descritas anteriormente, buscando mayor rentabilidad del sistema.

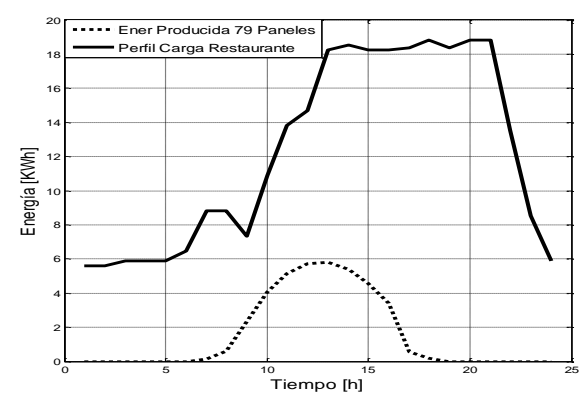

Fig. 2. Perfil de consumo de un restaurante vs energía suplida por 79 paneles 
Es claro entonces que se requiere de un sistema de almacenamiento para la energía que se desea redistribuir, el diseño de este dependerá tanto de la cantidad de energía a almacenar diariamente, como del rango del voltaje requerido por la referencia del inversor de voltaje seleccionado.

Dicho sistema estará conformado por un arreglo de baterías en serie y/o paralelo, dependiendo de los requerimientos, así, el número de baterías en serie dependerá del voltaje de entrada al inversor y el voltaje de cada batería del arreglo, como sigue. El número de baterías en paralelo indica el amperaje total que debe tener el arreglo para el almacenamiento de la energía.

\#Baterias Serie $=\frac{\text { Voltaje Inversor }[\mathrm{V}]}{\text { Voltaje Bateria[V] }}$

Capacidad Baterías $[\mathrm{Ah}]=\frac{\text { Energia a Almacenar }[\mathrm{Wh}]}{\text { Voltaje del Inversor }[\mathrm{V}]}$

La energía a almacenar se obtiene de la suma de la energía consumida en las horas valle de tarifa. Aplicando lo anterior al problema desarrollado hasta el momento, se pretende redistribuir la energía generada como se muestra en la Fig. 3.

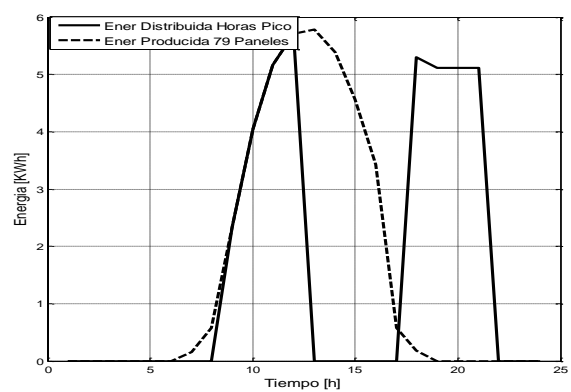

Fig. 3. Energía producida por 79 paneles vs energía distribuida en horas pico

Suponiendo que las baterías que se utilizarán en el sistema de almacenamiento trabajan a $12 \mathrm{~V}$ y que el inversor elegido lo hace a 
$180 \mathrm{~V}$, el número baterías requeridas para el presente sistema se obtiene de (17), así:

\#BateriasSerie $=15$

La capacidad de las baterías se calcula de la cantidad de energía que se requiere almacenar, que en este caso es $5.25 \mathrm{KWh}$. Reemplazando en (18) se obtiene una capacidad de 29.17 Ah. El diseño se reduce ahora a seleccionar la referencia de unas baterías que se ajusten a las anteriores especificaciones.

\section{ANÁLISIS DE COSTOS Y VIABILIDAD}

El análisis financiero pretende hacer un acercamiento más preciso acerca de la rentabilidad del sistema fotovoltaico, tiene en cuenta gran cantidad de variables que ilustran el comportamiento económico, con respecto al tiempo que tiene el mismo.

Para desarrollar el análisis financiero se realiza un flujo de fondos neto (Rojas, 2007) en un periodo de 20 años. El flujo de fondos se basa en diferentes variables, entre ellos, la inversión inicial, "La deducción por inversiones en control y mejoramiento del medio ambiente" (Monsalve, 2012), la depreciación de paneles y baterías, y los ingresos de cada periodo analizado, que en este caso en particular, corresponde al ahorro que representa suplir cierta cantidad de energía con los paneles solares y a la disminución de impuestos debido a la depreciación.

Se parte entonces, del análisis del ahorro en energía eléctrica mensual generado por la implementación del sistema fotovoltaico, tanto para el caso de implementar el sistema con paneles, como para en el caso de paneles más sistema de almacenamiento calculado en la sección anterior. Dicho análisis se ilustrará a partir del ejemplo a lo largo del artículo, para 79 paneles y 15 baterías en serie. El costo energía producida por los paneles es $\$ 399490$. El costo energía producida por los paneles+ baterías es de $\$ 404739$.

Como se observa en las Fig. 4 a y 4 b, el ahorro mensual por energía producida que representa el sistema de paneles más baterías es mayor. Ahora, es necesario determinar si este ahorro es 
suficiente para costear el valor de las baterías y dejar una utilidad extra para hacer el proyecto más rentable que en el primer caso (Únicamente paneles). Es fundamental para el análisis, señalar que la vida útil de las baterías está entre 6 y 8 años, mientras que la vida útil de los paneles se encuentra entre 20 y 25 años.

Ahora, partiendo del hecho de que la tarifa de energía eléctrica tiene un aumento de aproximadamente el 8\% anual (Según Empresas Públicas de Medellín), asumiendo la vida útil de las baterías y paneles de, 8 y 20 años respectivamente, además, suponiendo una renta líquida gravable del cliente de $\$ 150 ’ 000.000$ para todos los años de análisis y tomando una depreciación del 5\% anual para los paneles. Se puede hacer el flujo de fondos para ambos casos.

Para el caso ilustrado en la Fig. 5a, se tiene que el tiempo de retorno de inversión es 5 años, y el saldo después del periodo de análisis, 20 años, es de \$171'841.696. Claramente el proyecto es económicamente viable, dado que las ganancias superan ampliamente el valor de la inversión inicial que para este caso específico es de $\$ 16$ '306.970.

Similarmente, para el caso ilustrado en la Fig. 5b, que representa el flujo de fondos para el sistema con baterías, se tiene que el tiempo de retorno de inversión es 5 años, y el saldo después de 20 años es de $\$ 166$ '254.520. Nuevamente se obtiene que el proyecto es viable con una inversión inicial, y las particularidades anteriores, es alrededor de \$19'926.470.

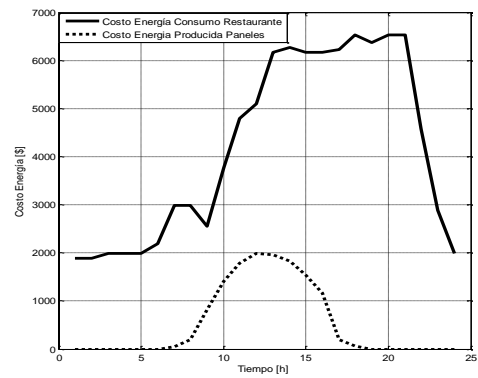

a)

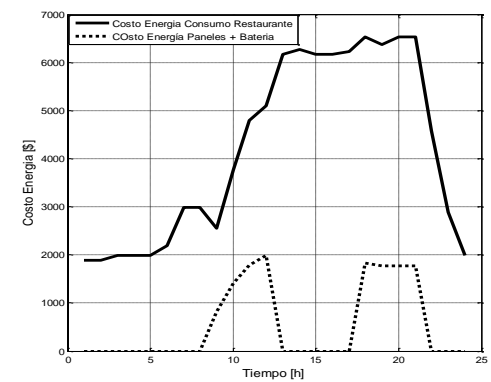

b)

Fig. 4. Costo de energía diario de un restaurante vs ahorro representado por a) 79 paneles y b) 79 paneles +15 baterías 
Se observa que, aunque los dos casos son viables, sigue siendo más rentable el caso de implementar solo los paneles, esto se debe a la corta vida útil de las baterías y el elevado costo que representa su reemplazo en este periodo.

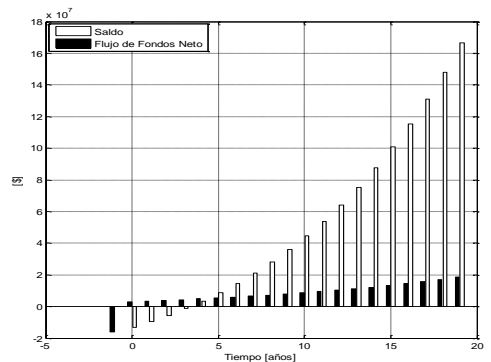

a)

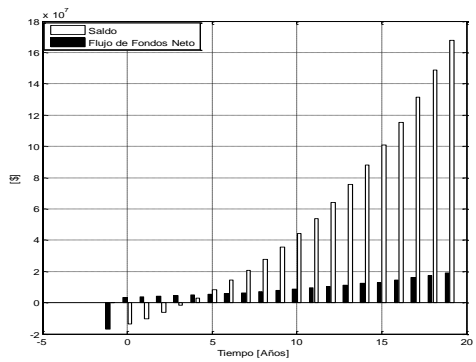

b)

Fig. 5. Flujo de fondos neto a 20 años, para un sistema de a) solo paneles y b) un sistema de paneles más baterías

Incluso si la viabilidad del uso de paneles más baterías fuera la misma que la del proyecto solo con paneles, la mejor opción será el proyecto sin baterías lpor simplicidad al ahorrar espacio y prescindir de horas de mantenimiento, además por factores ambientales como evitar la generación de desechos innecesarios ocasionados por el cambio del stock de baterías.

\section{CONCLUSIONES}

Si bien se observa que, de acuerdo con el costo de la energía producida, la implementación con baterías genera una mayor ganancia económica, la rentabilidad del sistema en general se condiciona con la inversión realizada en las baterías, tanto en su magnitud como en el número de veces que sea necesario realizarla, tomando como referencia el tiempo de vida de los paneles. En este orden de ideas, en el momento en el que el costo de las baterías se reduzca o bien, se aumente considerablemente su vida útil, la implementación del sistema de almacenamiento podrá ser replanteada como una opción más rentable. 
Independientemente del tipo específico de arreglo, ya sea solo paneles o paneles más sistema de almacenamiento, se pudo comprobar mediante el estudio financiero, la viabilidad económica de la implementación de un sistema fotovoltaico, basado en que las ganancias netas superan ampliamente la inversión inicial tomando 20 años que indican la vida útil aproximada de los paneles.

\section{AGRADECIMIENTOS}

Este trabajo ha sido desarrollado en la línea de investigación en Modelado y control de fuentes de potencia eléctrica del Grupo de Automática de la Universidad Nacional - GAUNAL, bajo los proyectos "MPPT Vectorial" y "Smart-Alen", en colaboración con el grupo de Automática y Electrónica del Instituto Tecnológico Metropolitano ITM.

\section{REFERENCIAS}

EPM. (2011, Mayo). Informe de tarifa y costos de energía eléctrica mercado regulado. www.epm.com

Kateeb, I. A., Bikdash, M., \& Chopade, P. (2011, March). Back to the future Renewable Energy Sources and green Smart Grid. In Southeastcon, 2011 Proceedings of IEEE (pp. 147-152). IEEE.

McLauchlan, L., \& Mehrubeoglu, M. (2010, April). A survey of green energy technology and policy. In Green Technologies Conference, 2010 IEEE (pp. 1-6). IEEE.

Monsalve, R. (2012). Impuestos 2012, estatuto tributario. Centro Interamericano Jurídico-financiero; Articulo 158-2.

Petrone, G., \& Ramos-Paja, C. A. (2011). Modeling of photovoltaic fields in mismatched conditions for energy yield evaluations. Electric Power Systems Research, 81(4), 1003-1013.

Rojas, M. (2007). Evaluación de proyectos para ingenieros. ECOE EDICIONES.

Universidad Nacional De Colombia, Facultad de ciencias. (2007). Caracterización del consumo de energía final en los sectores terciario, grandes establecimientos comerciales, centros comerciales y determinación de consumos para sus respectivos equipos de uso de energía final. pp. 259 . 